\title{
SAÚDE DA FAMÍLIA: CUIDADO NO DOMICÍLIO
}

\author{
Maria Rizoneide Negreiros de Araújo' \\ Luciana Carmem Sampaio ${ }^{2}$ \\ Maria Lígia Mohallem Carneiro ${ }^{3}$ \\ Roseni Rosângela de Sena ${ }^{4}$
}

\section{INTRODUÇÃO}

Os cuidados de saúde dispensados às pessoas nos domicílios não constituem uma prática nova. Os programas de saúde pública sempre enfatizaram ações de promoção e prevenção voltadas para grupos familiares em seus domicílios, quase sempre vinculados à atuação em atendimento à demandas específicas. Antes do surgimento dos grandes hospitais e da modalidade de assistência ambulatorial, no final do século XVIII, na Europa, os cuidados domiciliares já eram largamente empregados (Oliveira;Berger, 1996). Especificamente no campo da enfermagem, Florence

Os relatos de experiências discutidos no decorrer do curso, evidenciam que as demandas de internação domiciliar, a natureza dessas demandas e as condutas adotadas pelas equipes, carecem de respaldo, inclusive para diferenciá-la das visitas domiciliares e da própria internação hospitalar. Nightingale ao se referir à saúde como um estilo de vida afirmou, em 1863: "Cresce a convicção de que em todos os hospitais, por melhor que seja a sua administração, perdem-se vidas que poderiam ser poupadas; e que, via de regra, o pobre que recebesse boa assistência médica, cirúrgica e de enfermagem se recuperaria melhor na choça miserável em que vive do que no mais refinado ambiente hospitalar" (ABEn, 1982).

Independente do contexto vivenciado por Florence Nightingale, dos viéses histórico-ideológicos que permearam sua atuação, bem como as diferenças entre o ecossistema do século XIX e o do tempo atual, o que hoje se advoga no campo da saúde domiciliar já era preconizado, quando esta enfermeira deu início a revolução científica na enfermagem mundial.

Atualmente, em países como a Inglaterra, $20 \%$ das intervenções de saúde são prestadas fora das instituições de saúde (Oliveira;Berger, 1996).

No Brasil, a prestação de cuidados no domicílio não tem sido uma prática comum nas instituições de saúde públicas e privadas. Entretanto no início do século, a atividade prioritária no combate às grandes endemias que assolaram os grandes centros, eram as visitas domiciliares (Barreira, 1992). A partir deste fato, foi reconhecida a necessidade de formação de profissionais para este exercício. No entanto, esta formação era direcionada à prestação do cuidado para o doente ou para os fatores de risco de determinados agravos e não para a família como grupo social (Oliveira;Berger, 1996).

Com a institucionalização do Sistema Único de Saúde - SUS, a partir da Lei Federal no 8080/90 (BRASIL, 1990), estratégias vêm sendo pensadas e implementadas para a consolidação do seu modelo. Uma delas é a proposta do Ministério da Saúde, em 1994: o Programa de Saúde da Família PSF (FUNDAÇÃO NACIONAL DE SAÚDE, 1994). O PSF, na forma como foi concebido, surge como resposta às propostas de atenção primária de saúde que ocorreram na década de setenta e ao

\footnotetext{
${ }_{1}^{1}$ Professora Adjunta (aposentada) do Depto. Enfermagem Materno Infantil e Saúde Pública da Escola de Enfermagem da UFMG. Doutoranda em Enfermagem pela EEUSP.

${ }^{2}$ Aluna do 3o Período do Curso de Graduação da EE. UFMG. Bolsista de Iniciação Científica do CNPq.

${ }^{3}$ Professora Assistente II do Depto. de Enfermagem Materno Infantil e Saúde Pública da Escola de Enfermagem da UFMG. Doutoranda em Enfermagem pela EEUSP.

${ }^{4}$ Professora Adjunta I do Depto. de Enfermagem Materno Infantil e Saúde Pública da Escola de Enfermagem da UFMG. Doutora em Enfermagem pela EEUSP.
} 
movimento da Reforma Sanitária, que ocorreu na década de oitenta, culminando com a implantação do SUS. Neste contexto, a construção de um novo modelo assistencial embasado nas estratégias do PSF, propõe a organização das práticas de saúde voltadas para a atenção à família. Um dos pressupostos das estratégias do PSF elege a família e seu espaço social como o foco da atenção à saúde (MINISTÉRIO DA SAÚDE, 1996). Assim, o domicílio é considerado o cenário onde ocorrem as relações sociais geradoras de conflitos e de outros fatores de risco de adoecer, sendo também o local privilegiado para o desenvolvimento de ações de promoção e manutenção da saúde.

O Ministério da Saúde inclui dentre as atividades inerentes a atuação das equipes de saúde da família, a visita domiciliar. Ao se referir a mesma, agrega o componente internação domiciliar não como um substituto da internação hospitalar tradicional, e sim, como um recurso empregado com o "intuito de humanizar e garantir maior qualidade e conforto ao paciente" (MINISTÉRIO DA SAÚDE, 1997, p.14). Em acréscimo, destaca que a internação domiciliar só é indicada quando as condições clínicas e familiares do cliente forem favoráveis.

O cuidado dispensado à saúde no domicílio propicia à equipe de saúde da família, a inserção no cotidiano do cliente; identificando demandas e potencialidades da família, em um clima de parceria terapêutica. Para isso concorrem fatores como a humanização do cuidado, a ausência de riscos iatrogênicos de origem hospitalar, o resgate das formas de cuidar calcadas nas práticas tradicionalmente usadas pela população, embasadas na sua bagagem cultural.

Em relação ao custo desta atividade, considera-se que, embora seus custos possam, pela natureza da mesma, ser considerados onerosos, ela é um instrumento fundamental na educação para a saúde da população, além de propiciar melhoria da qualidade da atenção, oferecida com eqüidade. (Oliveira;Berger, 1996).

O contato com os profissionais enfermeiros e médicos integrantes das equipes de saúde da família, que freqüentam o Curso de Especialização em Saúde da Família ministrado pela Universidade Federal de Minas Gerais e pela Escola de Saúde de Minas Gerais, nos tem provocado inquietações. Os relatos de experiências discutidos no decorrer do curso, evidenciam que as demandas de internação domiciliar, a natureza dessas demandas e as condutas adotadas pelas equipes, carecem de respaldo, inclusive para diferenciá-la das visitas domiciliares e da própria internação hospitalar.

Buscando aproximação com a realidade desses profissionais, assim como contribuir para a construção de referenciais para uma "tão nova e tão antiga prática", optamos pela realização deste estudo, partindo das seguintes questões norteadoras:

- foram identificadas demandas de internação domiciliar no cotidiano de trabalho?

- qual a natureza destas demandas?

- quais os fatores favoráveis apontados?

- quais os fatores desfavoráveis apontados?

- Quais as principais condutas adotadas pelas equipes, nas situações que consideraram como internação domiciliar?

Essas questões nos levaram a estabelecer os objetivos que se seguem.

\section{OBJETIVOS}

- Identificar as demandas de internação domiciliar dos profissionais enfermeiros e médicos que integram equipes de saúde da família.

- Identificar os fatores favoráveis e desfavoráveis nas situações consideradas pelos profissionais como passíveis de internação domiciliar.

- Identificar as condutas adotadas pelos profissionais frente a situações consideradas como internação domiciliar.

\section{METODOLOGIA}

Trata-se de um estudo observacional, transversal e descritivo. O instrumento de coleta de da- 
dos utilizado foi um questionário com perguntas estruturadas relativas às questões norteadoras previamente estabelecidas para este estudo. Trabalhou-se com quarenta e um (41) profissionais enfermeiros e médicos de dezesseis (16) municípios do Estado de Minas Gerais, matriculados no Curso de Especialização em Saúde da Família, já mencionado.

TABELA 1 - Município de origem dos profissionais matriculados no curso de especialização em saúde da família, Belo Horizonte, 1998.

\begin{tabular}{l|c|c|c|c}
\hline \multirow{2}{*}{ MUNICÍPIOS } & \multicolumn{2}{|c|}{ PROFISSIONAIS } & TOTAL & $\%$ \\
\hline Abre Campo & Médicos & Enfermeiros & & \\
\hline Brumadinho & - & 01 & 01 & 2,4 \\
\hline Caeté & 01 & - & 01 & 2,4 \\
\hline Divinópolis & - & 03 & 03 & 7,3 \\
\hline Itambé do Mato & 04 & 02 & 06 & 14,6 \\
Dentro & 01 & - & 01 & \\
\hline Ibirité & 05 & - & 05 & 12,4 \\
\hline Itaúna & 01 & - & 01 & 2,4 \\
\hline Jaboticatubas & - & 01 & 01 & 2,4 \\
\hline Jequitibá & - & 01 & 01 & 2,4 \\
\hline Ouro Preto & 02 & 02 & 04 & 9,8 \\
\hline Ribeirão das Neves & - & 01 & 01 & 2,4 \\
\hline Santa Bárbara & 02 & 02 & 04 & 9,8 \\
\hline Santa Luzia & 04 & 03 & 07 & 17,2 \\
\hline São Gonçalo do & & & & \\
Pará & 01 & - & 01 & 2,4 \\
\hline Serra Azul & 01 & - & 01 & 2,4 \\
\hline Vespasiano & 01 & 18 & 41 & 100 \\
\hline TOTAL & 23 & & &
\end{tabular}

Dentre os respondentes, a maioria foi de médicos $(56,1 \%)$, sendo que $44 \%$ dos respondentes estão inscritos no PSF em municípios da região metropolitana de Belo Horizonte.

\section{ANÁLISE DOS RESULTADOS}

Quanto aos agravos passíveis de internação domiciliar (Tabela 2), na visão dos profissionais que responderam ao questionário, as doenças crônico-degenerativas foram as mais apontadas (31,7\%), seguidas das neoplasias (15\%) e da desidratação (15\%). Os cuidados pós-operatórios constituíram $3,3 \%$ e a situação de puerpério imediato totalizou $1,7 \%$ das respostas. A comparação entre os percentuais evidencia que a visão de internação domiciliar dos profissionais, pode estar mais centrada nas situações que exigem tratamento de doenças, do que na prevenção de iatrogenias decorrentes de situações que consideramos eletivas (pós-operatório e puerpério mediato). Os dados agrupados em outros correspondem a: alcoolismo, patologias do trato gênito-urinário, IRA, flebites e patologias osteoarticulares $(0,8 \%$ cada). 
TABELA 2 - Agravos e situações de internação domiciliar na visão dos profissionais - médicos e enfermeiros, como ação do programa de saúde da família. Belo Horizonte. 1998.

\begin{tabular}{l|c|c}
\hline \hline AGRAVOS & \multicolumn{2}{|c}{ Frequência } \\
\cline { 2 - 3 } & Abs. & $\%$ \\
\hline Doenças crônico-degenerativas & 38 & 31,6 \\
Neoplasias & 18 & 15,0 \\
Desidratação & 18 & 15,0 \\
Pneumonias & 15 & 12,5 \\
Diarréia & 09 & 7,5 \\
Cuidados pós-operatórios & 04 & 3,3 \\
Lesões medulares & 03 & 2,5 \\
Depressão & 02 & 1,7 \\
Desnutrição & 02 & 1,7 \\
Doenças gastro-intestinais & 02 & 1,7 \\
AIDS & 02 & 1,7 \\
Puerpério mediato & 02 & 1,7 \\
Outros & 05 & 4,1 \\
TOTAL & 120 & 100 \\
\hline \hline
\end{tabular}

Analisando os dados obtidos na Tabela 3, os relatos à vantagem de manter o cliente em seu próprio ambiente e da participação integrada dos familiares e clientes nos cuidados de saúde, totalizaram $41,2 \%$ das respostas, seguidos da diminuição do risco de infeção (13,9\%) e dos custos do tratamento $(10,3 \%)$. Destaques em relação à humanização da atenção $(2,2 \%)$, à diminuição do nível de ansiedade $(5,8 \%)$ e ao fortalecimento do vínculo cliente-família-equipe de saúde $(9,5 \%)$, constituíram 17,5\% das respostas. Isto nos leva a inferir que a visão dos profissionais tem aderência com os pressupostos da estratégia de atenção à saúde voltada para a saúde (MINISTÉRIO DA SAÚDE, 1996;1997).

TABELA 3 - Fatores favoráveis à internação domiciliar. Belo Horizonte, 1998.

\begin{tabular}{l|c|c}
\hline \hline FATORES FAVORÁVEIS & \multicolumn{2}{|c}{ Frequência } \\
\cline { 2 - 3 } & Abs. & $\%$ \\
\hline Mantém o paciente em seu próprio ambiente (casa) & 28 & 20,6 \\
Participação integrada de familiares e clientes nos cuidados à saúde & 28 & 20,6 \\
Menor risco de infecção & 19 & 14,0 \\
Tratamento menos oneroso & 14 & 10,3 \\
Fortalecimento do vínculo entre clientes e familiares com a equipe de & & \\
saúde da familia & 13 & 9,6 \\
Recuperação mais rápida do cliente & 10 & 7,4 \\
Diminuição da ansiedade do paciente frente a sua doença & 08 & 5,9 \\
Diminuição de internações & 06 & 4,5 \\
Atendimento humanizado & 03 & 2,2 \\
Qualidade da atenção & 02 & 1,4 \\
Aumento do sentimento de ser útil ao acompanhante familiar ou & & \\
profissional do PSF & 01 & 0,7 \\
Observação de outros fatores de risco não detectados & 01 & 0,7 \\
Acompanhamento diário do cliente & 01 & 0,7 \\
Orientação e supervisão freqüente dos cuidados de enfermagem & 01 & 0,7 \\
TOTAL & 136 & 100 \\
\hline \hline
\end{tabular}

Pelas informações contidas na Tabela 4, observa-se que a falta de equipamentos e de condições para a realização de cuidados pela equipe, destacou-se como o fator mais limitante da internação domiciliar (31,2\%). A falta de infra-estrutura e apoio logístico, tais como:

sistema de referência, disponibilidade de recursos diagnósticos complementares e de transporte, foi outro fator relevante como dificultados na realização de internação domiciliar (20\%).

A necessidade de capacitação dos profissionais para realizarem internação domiciliar ficou evidente com a freqüência de $15 \%$ de respostas indicativas da falta dessa capacitação.

A consideração da não permanência da equipe junto à família e ao cliente durante a noite e nos finais de semana como fator desfavorável (13,9\%), evidencia a incompreensão dos profissionais, não 
só no que diz respeito às diferenças entre internação domiciliar e internação domiciliar (MINISTÉRIO DA SAÚDE, 1997), como também do processo de trabalho inerente à equipe de saúde da família, que prevê maior acessibilidade população-equipe de saúde.

TABELA 4 - Fatores desfavoráveis à internação domiciliar.Belo Horizonte. 1998.

\begin{tabular}{l|c|c}
\hline \hline FATORES DESFAVORÁVEIS & \multicolumn{2}{|c}{ Frequência } \\
\cline { 2 - 3 } & Abs. & $\%$ \\
\hline Falta de equipamentos e condições para realização de procedimentos da & & \\
equipe de saúde & 25 & 31,3 \\
Falta de sistema de referência, tratamento e condição para realização de & & \\
exame laboratorial & 16 & 20,0 \\
Falta de profissional capacitado para atuar em internação domiciliar & 12 & 15,0 \\
Impossibilidade da equipe permanecer junto à família a noite e aos finais & & \\
de semana & 11 & 13,8 \\
Insegurança e desgaste emocional da família, cliente e da equipe de & & \\
saúde & 07 & 8,7 \\
Alteração na rotina da casa & 03 & 3,7 \\
Ingerência de terceiros & 02 & 2,5 \\
Outros & 04 & 5,0 \\
TOTAL & 80 & 100 \\
\hline \hline
\end{tabular}

TABELA 5 - Principais condutas adotadas pela equipe nas situações de internação domiciliar. Belo Horizonte, 1998.

\begin{tabular}{l|c|c}
\hline \hline CONDUTAS & \multicolumn{2}{|c}{ Frequência } \\
\cline { 2 - 3 } & Abs. & $\%$ \\
\hline Orientação da família & 15 & 18,1 \\
Visita diária da equipe de saúde & 14 & 16,9 \\
Cuidados no leito & 10 & 12,0 \\
Coletas de material para exame & 07 & 8,4 \\
Prescrições & 05 & 6,0 \\
Hidratação e medicação via oral & 05 & 6,0 \\
Venoclise & 05 & 6,0 \\
Medicação parenteral & 04 & 4,9 \\
Mudança de decúbito & 04 & 4,9 \\
Orientação dietética & 03 & 3,6 \\
Controle de sinais vitais & 02 & 2,4 \\
Tratamento de escaras & 02 & 2,4 \\
Curativos & 02 & 2,4 \\
Cateterismo vesical & 02 & 2,4 \\
Oxigenoterapia & 01 & 1,2 \\
Enteroclisma & 01 & 1,2 \\
Gavage & 01 & 1,2 \\
TOTAL & 83 & 100 \\
\hline \hline
\end{tabular}

A Tabela 5, na qual os respondentes apontaram as condutas adotadas nas situações que vivenciaram no seu cotidiano e consideraram como de internação domiciliar, aponta para o que consideramos como pertencentes à quatro agrupamentos de condutas:

- referentes à orientações diretas à família, não delegáveis, que dizem respeito a orientações gerais e dietéticas, correspondendo à $21,7 \%$;

- referentes à execução direta pela equipe, de procedimentos não delegáveis à família $(25,3 \%)$ que incluem visita diária pela equipe (16,9\%), prescrições $(6,0 \%)$, controle de sinais vitais $(2,4 \%)$. Em relação a essa última, cabe ressaltar que, embora a atividade possa ser orientada em sua execução pelo cliente ou família, não prescinde da aferição pela equipe de saúde;

- referentes à execução de procedimentos considerados a prioricomo de baixa complexidade $(27,7 \%)$ : cuidados no leito $(12,0 \%)$, hidratação e medicação via oral $(6,0 \%)$, mudanças de decúbito $(4,9 \%)$, tratamento de escaras $(2,4 \%)$ e curativos $(2,4 \%)$.

- referentes à procedimentos que exigem maior incorporação tecnológica $(25,3 \%)$ : coleta de 
ARAÚJO, Maria Rizoneide Negreiros de et al.

material para exame $(8,4 \%)$, venoclise $(6,0 \%)$, medicação parenteral $(4,9 \%)$, cateterismo vesical $(2,4 \%)$. Oxigenoterapia, enteroclisma e gavage corresponderam a 3,6\% do total de respostas.

\section{CONSIDERAÇÕES FINAIS}

A internação domiciliar ainda se constitui em um desafio para as equipes do Programa de Saúde da Família no Estado de Minas Gerais. O modelo de saúde vigente, em processo de transformação, carece de profundas revisões paradigmáticas, de modo que a doença deixe de ser o principal alvo da atenção, cedendo lugar à promoção da saúde e à prevenção de agravos.

A realização deste estudo, evidenciando que a visão dos profissionais enfermeiros e médicos privilegiou a internação domiciliar para portadores de doenças crônicas, coloca este procedimento como uma alternativa de desconcentração de serviços de saúde. Na possibilidade de dispensar aos clientes portadores de doenças crônicas, a mesma qualidade da atenção propiciada nos hospitais no que se refere ao atendimento das suas demandas, a internação domiciliar é considerada uma alternativa que favorece esta desconcentração. Entretanto há que se avaliar serem estas doenças crônicas de baixa gravidade, não necessitando de intervenções para reversão do quadro clínico, como por exemplo os clientes em fase terminal, os portadores de AIDS (Vaz, 1994).

Outra vantagem que o estudo mostrou foi a possibilidade de criação de um clima de parceria terapêutica na internação domiciliar. O cliente mantido no lar, a participação dos familiares e do próprio cliente nos cuidados, é acrescida pela diminuição de agravos iatrogênicos e a redução de custos no atendimento.

A falta de equipamentos adequados à internação domiciliar e outras desvantagens apontadas pelo grupo estudado (ausência dos profissionais nos finais de semana, falta de apoio logístico, entre outras) nos leva a considerar que:

- a internação domiciliar como estratégia de desconcentração dos serviços de saúde, de humanização da atenção e de co-participação no cuidado do cliente, carece de criação de protocolos que norteem o trabalho dos profissionais;

- o eixo destes protocolos, não restritos à abordagem clínica do cliente em internação domiciliar, devem contemplar ações que considerem a família como espaço social privilegiado para o desenvolvimento de ações de proteção e promoção da saúde.

\section{REFERÊNCIAS BIBLIOGRÁFICAS}

\section{ASSOCIAÇÃO BRASILEIRA DE ENFERMAGEM. Boletim Informativo. Brasília, DF. mai. 1982. s.n.t.}

BARREIRA, I. de A. A enfermeira-Ananéri no "País do Futuro": a aventura de luta contra a tuberculose. Rio de Janeiro, 1992. 355 p. Tese. (Doutoramento em Enfermagem) - Escola de Enfermagem Anna Nery, Universidade Federal do Rio de Janeiro.

BRASIL. Lei n 8080 de 19/09/1990. Dispõe sobre as condições para a formação, proteção e recuperação da saúde, da organização e funcionamento dos serviços correspondentes e de outras providências. Diário Oficial da União, 20 de setembro de 1990.

FUNDAÇÃO NACIONAL DE SAÚDE. Departamento de Operações. Coordenação de Saúde da Comunidade. Programa Saúde da Família. Brasília: Ministério da Saúde, 1994.

MINISTÉRIO DA SAÚDE. Secretaria de Assistência à Saúde. Departamento de Assistência e Promoção à Saúde. Coordenação de Saúde da Comunidade. Saúde da Família: uma estratégia de organização dos serviços de saúde. Brasília, 1996. (Doc. Preliminar).

OLIVEIRA, F.J.A. de; BERGER, C.B. Visitas domiciliares em atenção primária à saúde: eqüidade e qualidade dos serviços. Mom. \& Perspec. Saúde. Porto Alegre, v. 9, n. 2, jul./dez. 1996.

VAZ, J.C. Assistência domiciliar à saúde. Dicas - idéias para a ação municipal. Secretaria Municipal de Saúde. Santos. SP, n.8, mar. 1994. 